\title{
Robust Utility Maximization in a Stochastic Factor Model
}

\section{Daniel Hernández-Hernández*} Alexander Schied**

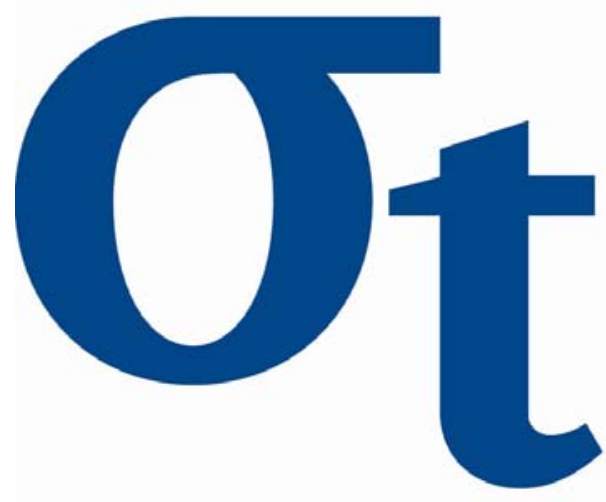

* Centro de Investigación en Matemáticas, Guanajuato, México ** Institute of Mathematics, Technische Universität Berlin, Germany

This research was supported by the Deutsche Forschungsgemeinschaft through the SFB 649 "Economic Risk". 


\title{
Robust utility maximization in a stochastic factor model
}

\author{
Daniel Hernández-Hernández and Alexander Schied
}

August 30, 2006

\begin{abstract}
We give an explicit PDE characterization for the solution of a robust utility maximization problem in an incomplete market model, whose volatility, interest rate process, and long-term trend are driven by an external stochastic factor process. The robust utility functional is defined in terms of a HARA utility function with negative risk aversion and a dynamically consistent coherent risk measure, which allows for model uncertainty in the distributions of both the asset price dynamics and the factor process. Our method combines two recent advances in the theory of optimal investments: the general duality theory for robust utility maximization and the stochastic control approach to the dual problem of determining optimal martingale measures.
\end{abstract}

\section{Introduction}

One of the fundamental problems in mathematical finance is the construction of investment strategies that maximize the utility functional of a risk-averse investor. In the vast majority of the corresponding literature it is assumed that the optimality criterion is based on a classical expected utility functional of the form

$$
X \longmapsto \mathbb{E}[U(X)]
$$

where $U$ is a utility function. This concept involves the expected value with respect to the probability measure $\mathbb{P}$, which is usually assumed to model accurately future stock price evolutions. In reality, however, the choice of this probability measure is subject to model risk, and it may thus be reasonable to replace the expectation operator in (1) by (the negative of) a coherent risk measure, thus obtaining a robust utility functional of the form

$$
X \longmapsto \inf _{Q \in \mathcal{Q}} E_{Q}[U(X)]
$$

cf. Schmeidler [23] and Gilboa and Schmeidler [14]. See also Föllmer and Schied [13, 12] for the relations with coherent risk measures, and Maccheroni et al. [17] for a recent extension to the case of convex risk measures.

AMS 2000 subject classification: 91B28, 49L20, 90C47, 60H10

Key words and phrases: optimal investment, model uncertainty, incomplete markets, stochastic volatility, coherent risk measures, optimal control, convex duality 
Optimal investment problems for robust utility amount to the maximization of functionals (2) over the set of possible payoffs arising from admissible trading strategies. Such problems were considered, among others, by Talay and Zheng [?], Quenez [19], Schied [20, 21], Burgert and Rüschendorf [4], Schied and Wu [22], Müller [18], and Föllmer and Gundel [11]. See also Hansen and Sargent [15] and Bordigoni et al. [3] for the analysis of a related problem involving entropic penalties. Most of these papers use either the duality method (sometimes also called the 'martingale method') [11, 19, 21, 22] or stochastic control techniques based on backward stochastic differential equations [3, 18, 19]. Talay and Zheng [?] apply a PDE-based control approach directly to the primal maximin problem and obtain a characterization of the value function as viscosity solution of a Hamilton-Jacobi-Bellman-Isaacs equation with a game-type nonlinearity.

In this paper, we will present a new approach that consists in combining the duality results from $[21,22]$ with a stochastic control approach to the dual problem of determining optimal martingale measures. This stochastic control approach was recently developed by Castañeda-Leyva and Hernández-Hernández [5, 6] for utility maximization problems in incomplete financial market models, whose volatility, interest rate process, and trend are driven by an external stochastic factor process. The basic idea in $[5,6]$ is to derive a Hamilton-Jacobi-Bellmann PDE for the dual value function, which involves the 'risk premia' of equivalent local martingale measures as control processes. Already in standard utility maximization problems, this approach turned our to be very powerful as it provides an explicit characterization of optimal strategies in terms of the unique classical solution of a nonlinear PDE, which then can be solved numerically. As for robust utility maximization, it was already observed by Quenez [19] that it is natural to apply control methods to the dual problem rather than to the primal one, since the dual value function $v$ of the robust problem has a much simpler structure than the primal value function $u$ : The function $v$ is defined in terms of an infimum taken over a two-parameter set, while $u$ involves an infimum with respect to one and a supremum with respect to another parameter.

In setting up our model, we will use the framework of $[5,6]$ to set up our reference model and then suppose that the dynamics of both the asset prices and the stochastic factor process are subject to model uncertainty. To this end, we have to specify the prior set $\mathcal{Q}$ occurring in the representation (2) of the robust utility functional. While the duality method works for very general prior sets, the use of control techniques requires the restriction to classes $\mathcal{Q}$ that satisfy a property of dynamic consistency as described, e.g., by Artzner et al. [2], Delbaen [7], and Epstein and Schneider [8]. We also need to work with a very specific utility function, namely a HARA utility function

$$
U(x)=\frac{1}{\gamma} x^{\gamma}
$$

with risk aversion parameter $\gamma<0$. The cases $\gamma=0$ and $\gamma>0$ are also feasible but require different methods, so that they will be treated elsewhere.

This loss of generality in comparison with the duality method will be rewarded by much more specific results, which are apt to explicit numerical computations. More precisely, 
our main result provides explicit formulas for both the optimal strategy and the robust value function in terms of the unique bounded classical solution of a nonlinear PDE. In particular, we avoid the use of viscosity solutions. As a byproduct, we also obtain a formula for the least-favorable martingale measure in the sense of Föllmer and Gundel [11].

This paper is organized as follows. In Section 2 we describe the set-up of the problem and state a theorem containing our main findings. This theorem will be proved in the subsequent sections. The dual problem for our robust utility maximization problem is formulated in Section 3. In Section 4 we derive a Hamilton-Jacobi-Bellman PDE for the value function of the dual problem. In Section 5 we finally get back to the primal problem and show how the optimal investment strategy can be derived from our solution to the dual problem.

\section{Statement of main results}

We consider a financial market model with a locally riskless money market account

$$
d S_{t}^{0}=S_{t}^{0} r\left(Y_{t}\right) d t
$$

and a risky asset defined under a reference measure $\mathbb{P}$ through the SDE

$$
d S_{t}=S_{t} b\left(Y_{t}\right) d t+S_{t} \sigma\left(Y_{t}\right) d W_{t}^{1}
$$

Here $W^{1}$ is a standard $\mathbb{P}$-Brownian motion and $Y$ denotes an external economic factor process modeled by the SDE

$$
d Y_{t}=g\left(Y_{t}\right) d t+\rho d W_{t}^{1}+\sqrt{1-\rho^{2}} d W_{t}^{2}
$$

for some correlation factor $\rho \in[-1,1]$ and a standard $\mathbb{P}$-Brownian motion $W^{2}$, which is independent of $W^{1}$ under $\mathbb{P}$. We suppose that the economic factor cannot be traded directly so that the market model is typically incomplete. It will be convenient to use the shorthand notation

$$
\bar{\rho}:=\sqrt{1-\rho^{2}} .
$$

We assume that $g(\cdot)$ is in $C^{1}(\mathbb{R})$, with derivative $g^{\prime} \in C_{b}^{1}(\mathbb{R})$, and $r(\cdot), b(\cdot)$, and $\sigma(\cdot)$ belong to $C_{b}^{2}(\mathbb{R})$, where $C_{b}^{k}(\mathbb{R})$ denotes the class of bounded functions with bounded derivatives up to order $k$. The assumption of time-independent coefficients is for notational convenience only and can easily be relaxed. The 'market price of risk' is defined via the function

$$
\theta(y):=\frac{b(y)-r(y)}{\sigma(y)},
$$

and we will assume that $\sigma(\cdot) \geq \sigma_{0}>0$ for some constant $\sigma_{0}$.

In most economic situations, investors typically face model uncertainty in the sense that the dynamics of the relevant quantities are not precisely known. One common 
approach to coping with model uncertainty is to admit an entire class $\mathcal{Q}$ of possible prior models; see, e.g., [13, Section 2.5]. Here, we will consider the class

$$
\mathcal{Q}:=\left\{Q \sim \mathbb{P} \mid \frac{d Q}{d \mathbb{P}}=\mathcal{E}\left(\int \eta_{1 t} d W_{t}^{1}+\int \eta_{2 t} d W_{t}^{2}\right)_{T}, \eta=\left(\eta_{1}, \eta_{2}\right) \in \mathcal{C}\right\},
$$

where $\mathcal{E}(M)_{t}=\exp \left(M_{t}-\langle M\rangle_{t} / 2\right)$ denotes the Doleans-Dade exponential of a local martingale $M$ and $\mathcal{C}$ denotes the set of all progressively measurable processes $\eta=\left(\eta_{1}, \eta_{2}\right)$ such that $\eta_{t}$ belongs $d t \otimes d \mathbb{P}$-a.e. to some fixed compact convex set $\Gamma \subset \mathbb{R}^{2}$. Note that due to Novikov's theorem we have a one-to-one correspondence between measures $Q \in \mathcal{Q}$ and processes $\eta \in \mathcal{C}$ (up to $d t \otimes d \mathbb{P}$-nullsets).

For a progressively measurable process $\pi$ such that $\int_{0}^{T} \pi_{s}^{2} d s<\infty \mathbb{P}$-a.s.,

$$
\begin{aligned}
X_{t}^{x, \pi} & =x+\int_{0}^{t} \frac{X_{s}^{x, \pi}\left(1-\pi_{s}\right)}{S_{s}^{0}} d S_{s}^{0}+\int_{0}^{t} \frac{X_{s}^{x, \pi} \pi_{s}}{S_{s}} d S_{s} \\
& =x+\int_{0}^{t} X_{s}^{\pi}\left(r\left(Y_{s}\right)+\left[b\left(Y_{s}\right)-r\left(Y_{s}\right)\right] \pi_{s}\right) d s+\int_{0}^{T} X_{s}^{\pi} \pi_{s} \sigma\left(Y_{s}\right) d W_{s}^{1}
\end{aligned}
$$

describes the evolution of the wealth process $X^{x, \pi}$ of an investor with initial endowment $X_{0}^{x, \pi}=x>0$ investing the fraction $\pi_{s}$ of the current wealth into the risky asset at time $s \in[0, T]$. The strategy $\pi$ is called admissible at level $x$ if $X^{x, \pi} \geq 0$, and we denote by $\mathcal{A}(x)$ the set of all such strategies.

The objective of the investor consists in

$$
\text { maximizing } \inf _{Q \in \mathcal{Q}} E_{Q}\left[U\left(X_{T}^{x, \pi}\right)\right] \text { over } \pi \in \mathcal{A}(x),
$$

where the utility function $U:] 0, \infty[\rightarrow \mathbb{R}$ will be specified in the sequel as a HARA utility function

$$
U(x)=\frac{x^{\gamma}}{\gamma} \quad \text { with risk aversion parameter } \gamma<0 .
$$

As already menioned in the introduction, the cases $\gamma=0$ and $\gamma>0$ are also feasible but require different methods and will be discussed elsewhere. We summarize our main findings in the following theorem.

Theorem 2.1 The value function of the robust utility maximization problem (7) is given by

$$
u(x):=\sup _{\pi \in \mathcal{A}(x)} \inf _{Q \in \mathcal{Q}} E_{Q}\left[U\left(X_{T}^{x, \pi}\right)\right]=\frac{1}{\gamma} x^{\gamma} e^{(1-\gamma) w\left(0, Y_{0}\right)},
$$

where $w:[0, T] \times \mathbb{R} \rightarrow \mathbb{R}$ is the unique bounded classical solution of the nonlinear PDE

$$
\begin{aligned}
0=w_{t}+\frac{1}{2} w_{y y} & +(g-\alpha \rho \theta) w_{y}+\frac{1}{2} \cdot \frac{1-\alpha \rho^{2}}{1-\alpha} w_{y}^{2}-\alpha r+ \\
& +\max _{\eta \in \Gamma}\left[\rho(1-\alpha) \eta_{1} w_{y}-\frac{\alpha(1-\alpha)}{2}\left(\theta+\eta_{1}\right)^{2}+\eta_{2} \bar{\rho} w_{y}\right]
\end{aligned}
$$

with terminal condition

$$
w(T, \cdot) \equiv 0
$$


and $\alpha:=-\gamma /(1-\gamma)$. If the $\Gamma$-valued function $\eta^{*}=\eta^{*}(t, y)$ realizes the maximum in $(9)$, then an optimal strategy $\widehat{\pi}$ for the robust problem can be obtained by letting $\widehat{\pi}_{t}=\pi^{*}\left(t, Y_{t}\right)$ for

$$
\pi^{*}(t, y)=\frac{1}{\sigma(y)}\left[(1-\alpha)\left(\eta_{1}^{*}(t, y)+\theta(y)\right)+\rho w_{y}(t, y)\right] .
$$

Moreover, by defining a measure $\widehat{Q} \in \mathcal{Q}$ via

$$
\frac{d \widehat{Q}}{d \mathbb{P}}=\mathcal{E}\left(\int_{0} \eta_{1}^{*}\left(t, Y_{t}\right) d W_{t}^{1}+\int_{0} \eta_{2}^{*}\left(t, Y_{t}\right) d W_{t}^{2}\right)_{T}
$$

we obtain a saddlepoint $(\widehat{\pi}, \widehat{Q})$ for the maximin problem $(7)$.

Remark 2.2 If the coefficients $b$ and $\sigma$ are constant, then the value function $u$ will clearly not depend on $Y_{0}$. Hence, $w$ will be constant and $w_{y}$ will vanish. Determining the optimal $\eta^{*}$ will thus be reduced to finding the value $\eta_{1}^{*}$ closest to $\theta$. We hence recover a particular case of the results in [19, Section 7.5] and [20, Section 3.1]. A similar situation occurs if $\rho=0$ and $\Gamma$ is a rectangle: it will again be optimal to minimize the distance between $\eta_{1}^{*}$ and $\theta$. In particular, $\widehat{Q}$ will locally be a martingale measure and our formula for $\pi^{*}$ shows that there will be no investment into the risky asset as long as the factor process $Y$ stays in the region

$$
\mathcal{N}:=\left\{y \in \mathbb{R} \mid\left(-\theta(y), \eta_{2}\right) \in \Gamma \text { for some } \eta_{2} \in \mathbb{R}\right\} .
$$

A nonzero correlation factor $\rho$, however, can change the picture. More precisely, let us assume that the factor $\rho w_{y}$ is nonzero on $\mathcal{N}$, which seems to be plausible provided that $Y$ can exit $\mathcal{N}$ with positive probability. In this case, our formula for $\pi^{*}$ shows that even for $Y_{t} \in \mathcal{N}$ there will be a nontrivial investment into the risky asset - despite the fact that we can turn discounted asset prices locally into a martingale by choosing an appropriate $Q \in \mathcal{Q}$. This effect occurs as a tradeoff between the tendencies of minimizing asset returns and driving $Y$ further away from 'favorable regions' under the 'worst-case measure' $\widehat{Q}$. It could be interesting to see this intuition confirmed by numerical experiments.

Remark 2.3 As a byproduct of our proof, we also obtain an explicit formula for a least favorable martingale measure $P^{*}$ as considered by Föllmer and Gundel [11]. It is associated to our optimal strategy via the formula

$$
\frac{d P^{*}}{d \mathbb{P}}=\mathcal{E}\left(-\int \theta\left(Y_{s}\right) d W_{s}^{1}-\int \widehat{\nu}_{s} d W_{s}^{2}\right)_{T}
$$

where

$$
\widehat{\nu}_{t}=-\eta_{2}^{*}\left(t, Y_{t}\right)-\frac{\bar{\rho}}{1-\alpha} \cdot w_{y}\left(t, Y_{t}\right)
$$

\section{Formulation of the dual problem}

In this section, we will first use robust duality theory as to reduce the solution of our original problem to its dual problem. The dual problem will then be solved by stochastic 
control techniques in Section 4. The duality theory for robust utility maximization problems of the form (7) was developed by Quenez [19], Schied and Wu [22], and Schied [21]. Utility functions of the form (8) are ruled out by [19, Assumption 5.1], and so we will rely on $[22,21]$ as our sources of reference on duality.

To check for the applicability of the results in [22, 21], note first that our utility function (8) belongs to $C^{1}$, is increasing and strictly concave, and satisfies the Inada conditions $U^{\prime}(0+)=\infty$ and $U^{\prime}(\infty-)=0$. It also has asymptotic elasticity $A E(U)=$ $\lim \sup _{x \uparrow \infty} x U^{\prime}(x) / U(x)=0<1$. Moreover, our prior set $\mathcal{Q}$ satisfies [22, Assumption 2.1]:

Lemma 3.1 The set $\{d Q / d \mathbb{P} \mid Q \in \mathcal{Q}\}$ is convex and closed in $L^{0}(\mathbb{P})$.

Proof: Let us introduce the notation

$$
D_{t}^{\eta}:=\mathcal{E}\left(\int_{0} \eta_{1 s} d W_{s}^{1}+\int_{0} \eta_{2 s} d W_{s}^{2}\right)_{t} \quad \text { for } \eta \in \mathcal{C} .
$$

To show convexity, we take $0 \leq \lambda \leq 1$ and $\eta, \widetilde{\eta} \in \mathcal{C}$. Following Delbaen [7], we see that the martingale $D:=\lambda D^{\eta}+(1-\lambda) D^{\widetilde{\eta}}$ satisfies the $\operatorname{SDE} d D_{t}=D_{t}\left(\xi_{1 t} d W_{t}^{1}+\xi_{2 t} d W_{t}^{2}\right)$, where $\xi_{t}=\alpha_{t} \eta_{t}+\left(1-\alpha_{t}\right) \widetilde{\eta}_{t}$ is at each time a convex combination of $\eta$ and $\widetilde{\eta}$ with coefficient

$$
\alpha_{t}=\frac{\lambda D_{t}^{\eta}}{\lambda D_{t}^{\eta}+(1-\lambda) D_{t}^{\widetilde{\eta}}}
$$

Hence, $\xi$ takes values in $\Gamma$ and belongs to $\mathcal{C}$.

To prove the closedness assertion, note first that, for any $p \in \mathbb{R}$,

$$
\mathbb{E}\left[\left(D_{T}^{\eta}\right)^{p}\right]=\mathbb{E}\left[D_{T}^{p \eta} \cdot e^{\frac{1}{2} p(p-1) \int_{0}^{T}\left|\eta_{t}\right|^{2} d t}\right] \leq e^{\frac{1}{2}\left(p^{2}+|p|\right) T \sup _{\gamma \in \Gamma}|\gamma|^{2}}<\infty .
$$

Thus, if $D_{T}^{\eta^{n}}$ converges in probability to some random variable $D \in L^{0}(\mathbb{P})$, then both $D_{T}^{\eta^{n}}$ and $\left(D_{T}^{\eta^{n}}\right)^{-1}$ converge in $L^{p}(\mathbb{P})$ for any $p \geq 1$. It thus follows easily that the stochastic integrals $\int_{0}^{T}\left(D_{t}^{\eta^{n}}\right)^{-1} d D_{t}^{\eta^{n}}$ form a Cauchy sequence in $L^{2}(\mathbb{P})$. Now the result follows from the fact that

$$
\mathbb{E}\left[\left(\int_{0}^{T} \frac{1}{D_{t}^{\eta^{n}}} d D_{t}^{\eta^{n}}-\int_{0}^{T} \frac{1}{D_{t}^{\eta^{k}}} d D_{t}^{\eta^{k}}\right)^{2}\right]=\mathbb{E}\left[\int_{0}^{T}\left|\eta_{t}^{n}-\eta_{t}^{k}\right|^{2} d t\right]
$$

Let us denote by $\mathcal{M}$ the set of all progressively measurable processes $\nu$ such that $\int_{0}^{T} \nu_{t}^{2} d t<\infty \mathbb{P}$-a.s., and define

$$
Z_{t}^{\nu}:=\mathcal{E}\left(-\int \theta\left(Y_{s}\right) d W_{s}^{1}-\int \nu_{s} d W_{s}^{2}\right)_{t}
$$

Then one easily shows that $Z_{t}^{\nu} X_{t}^{x, \pi} / S_{t}^{0}$ is a positive local $\mathbb{P}$-martingale and hence a $\mathbb{P}$ supermartingale for all $\nu \in \mathcal{M}$ and $\pi \in \mathcal{A}(x)$. That is, every process $Z^{\nu}$ belongs to the class $\mathcal{Y}(1)$ as defined in [16] and further considered in a robust framework in [22, 21]. 
Moreover, the density process of every equivalent local martingale measure is of the form $Z^{\nu}$ for some $\nu \in \mathcal{M}$. Hence, it follows from [16, Theorem 2.2] that the dual value function with subjective measure $\mathbb{P}$ is given by

$$
\widetilde{u}_{\mathbb{P}}(\lambda)=\inf _{\nu \in \mathcal{M}} \mathbb{E}\left[\widetilde{U}\left(\lambda Z_{T}^{\nu} / S_{T}^{0}\right)\right], \quad \lambda>0,
$$

where $\widetilde{U}(z)=\sup _{x \geq 0}(U(x)-z x)$ is the Fenchel-Legendre transform of the convex function $-U(-x)$. If we use instead of $\mathbb{P}$ another subjective measure $Q \in \mathcal{Q}$ with density $D:=$ $d Q / d \mathbb{P}$, then the corresponding dual value function is of the form

$$
\widetilde{u}_{Q}(\lambda)=\inf _{\nu \in \mathcal{M}} \mathbb{E}\left[D \widetilde{U}\left(\lambda Z_{T}^{\nu} /\left(D S_{T}^{0}\right)\right)\right] .
$$

It thus follows from [22, Theorems 2.2 and 2.6] that the dual value function of the robust utility maximization problem is given as

$$
\widetilde{u}(\lambda):=\inf _{Q \in \mathcal{Q}} \widetilde{u}_{Q}(\lambda)=\inf _{\eta \in \mathcal{C}} \inf _{\nu \in \mathcal{M}} \mathbb{E}\left[D_{T}^{\eta} \widetilde{U}\left(\frac{\lambda Z_{T}^{\nu}}{D_{T}^{\eta} S_{T}^{0}}\right)\right],
$$

where $D^{\eta}$ is as in (11). Due to [22, Theorem 2.2], the primal value function

$$
u(x):=\sup _{\pi \in \mathcal{A}(x)} \inf _{Q \in \mathcal{Q}} E_{Q}\left[U\left(X_{T}^{x, \pi}\right)\right]
$$

can then be obtained as

$$
u(x)=\min _{\lambda>0}(\widetilde{u}(\lambda)+\lambda x) .
$$

Moreover, if there are $(\widehat{\eta}, \widehat{\nu})$ control processes minimizing (12), then [21, Theorem 2.6] yields the existence of an optimal strategy $\widehat{\pi} \in \mathcal{A}(x)$, whose terminal wealth is given by

$$
X_{T}^{x, \widehat{\pi}}=I\left(\frac{\widehat{\lambda} Z_{T}^{\widehat{\nu}}}{D_{T}^{\hat{v}} S_{T}^{0}}\right),
$$

where $I(y):=-\widetilde{U}^{\prime}(y)$ and $\widehat{\lambda}>0$ minimizes (13).

In our specific setting (8), we have

$$
\widetilde{U}(z)=-\frac{z^{\alpha}}{\alpha} \quad \text { with } \alpha=\frac{-\gamma}{1-\gamma} .
$$

Note that $0<\alpha<1$. Thus, we can simplify the duality formula (13) as follows. First, the expectation in (12) can be computed as

$$
\mathbb{E}\left[D_{T}^{\eta} \widetilde{U}\left(\frac{\lambda Z_{T}^{\nu}}{D_{T}^{\eta} S_{T}^{0}}\right)\right]=-\frac{\lambda^{\alpha}}{\alpha} \mathbb{E}\left[\left(D_{T}^{\eta}\right)^{1-\alpha}\left(Z_{T}^{\nu}\right)^{\alpha}\left(S_{T}^{0}\right)^{-\alpha}\right]=:-\frac{\lambda^{\alpha}}{\alpha} \Lambda_{\eta, \nu} .
$$

Optimizing over $\lambda \geq 0$ then yields that

$$
\min _{\lambda \geq 0}\left(-\frac{\lambda^{\alpha}}{\alpha} \Lambda_{\eta, \nu}+\lambda x\right)=\frac{\alpha-1}{\alpha} x^{-\alpha /(1-\alpha)} \Lambda_{\eta, \nu}^{1 /(1-\alpha)}=\frac{x^{\gamma}}{\gamma} \Lambda_{\eta, \nu}^{1-\gamma},
$$


where the optimal $\lambda$ is given by

$$
\widehat{\lambda}=\left(\frac{\Lambda_{\eta, \nu}}{x}\right)^{1 /(1-\alpha)} .
$$

Using (12) and (13) now yields

$$
u(x)=\frac{x^{\gamma}}{\gamma}\left(\sup _{\nu \in \mathcal{M}} \sup _{\eta \in \mathcal{C}} \Lambda_{\eta, \nu}\right)^{1-\gamma}
$$

To further simplify $\Lambda_{\eta, \nu}$, note that

$$
\begin{aligned}
& \left(D_{T}^{\eta}\right)^{1-\alpha}\left(Z_{T}^{\nu}\right)^{\alpha}\left(S_{T}^{0}\right)^{-\alpha} \\
& =\mathcal{E}\left(\int\left((1-\alpha) \eta_{1 t}-\alpha \theta\left(Y_{t}\right)\right) d W_{t}^{1}+\int\left((1-\alpha) \eta_{2 t}-\alpha \nu_{t}\right) d W_{t}^{2}\right)_{T} \\
& \quad \times \exp \left(\int_{0}^{T} q\left(Y_{t}, \eta_{t}, \nu_{t}\right) d t\right)
\end{aligned}
$$

where the function $q: \mathbb{R} \times \mathbb{R}^{2} \times \mathbb{R} \rightarrow \mathbb{R}$ is given by

$$
q(y, \eta, \nu)=-\frac{\alpha(1-\alpha)}{2}\left[\left(\eta_{1}+\theta(y)\right)^{2}+\left(\eta_{2}+\nu\right)^{2}\right]-\alpha r(y) .
$$

The Doleans-Dade exponential in (16) will be denoted by $\Delta_{T}^{\eta, \nu}$. If $\int_{0}^{T} \nu_{t}^{2} d t$ is bounded, then $\mathbb{E}\left[\Delta_{T}^{\eta, \nu}\right]=1$. We thus define

$$
\mathcal{M}_{0}:=\left\{\nu \in \mathcal{M} \mid \int_{0}^{T} \nu_{t}^{2} d t \text { is } \mathbb{P} \text {-a.s. bounded }\right\} .
$$

Lemma 3.2 For fixed $\eta \in \mathcal{C}$ we have

$$
\sup _{\nu \in \mathcal{M}} \Lambda_{\eta, \nu}=\sup _{\nu \in \mathcal{M}_{0}} \Lambda_{\eta, \nu}
$$

Proof: For $\nu \in \mathcal{M}$ given let $\tau_{n}:=\inf \left\{t \geq 0 \mid \int_{0}^{t} \nu_{s}^{2} d s \geq n\right\} \wedge T$. Then $\nu_{t}^{n}:=\nu_{t} \mathrm{I}_{\left\{\tau_{n}>t\right\}}$ belongs to $\mathcal{M}_{0}$ and $Z_{T}^{\nu^{n}}$ converges $\mathbb{P}$-a.s. to $Z_{T}^{\nu}$ as $n \rightarrow \infty$. Moreover, the negative parts of the sequence

$$
D_{T}^{\eta} \widetilde{U}\left(\frac{Z_{T}^{\nu^{n}}}{D_{T}^{\eta} S_{T}^{0}}\right)=\frac{-1}{\alpha}\left(D_{T}^{\eta}\right)^{1-\alpha}\left(Z_{T}^{\nu^{n}}\right)^{\alpha}\left(S_{T}^{0}\right)^{-\alpha}, \quad n \in \mathbb{N},
$$

are uniformly $\mathbb{P}$-integrable according to $[22$, Lemma 3.6]. But under our assumption $\gamma<0, \widetilde{U}$ takes only negative values and we obtain that $\Lambda_{\eta, \nu^{n}}$ converges to $\Lambda_{\eta, \nu}$. 


\section{HJB solution of the dual problem}

In this section, we will solve the dual problem by stochastic control techniques. Here we rely on the methods developed by Castañeda-Leyva and Hernández-Hernández [5, 6], and we will extend them to our robust setting.

Our aim is to maximize $\Lambda_{\eta, \nu}$ over $\eta \in \mathcal{C}$ and $\nu \in \mathcal{M}_{0}$. To this end, let us now consider a starting time $t \in[0, T]$ replacing our previous choice $t=0$. This will be formalized by introducing the measure $\mathbb{P}_{t, y}$ under which the processes $\left(S_{u}^{0}\right)_{u \geq t},\left(S_{u}\right)_{u \geq t}$, and $\left(Y_{u}\right)_{u \geq t}$ satisfy their respective stochastic differential equations (3), (4), and (5) with initial conditions

$$
S_{t}^{0}=1, S_{t}=\text { arbitrary, and } Y_{t}=y .
$$

Also, under $\mathbb{P}_{t, y}$ all stochastic exponentials will only involve martingale increments from time $t$ onwards, e.g.,

$$
Z_{T}^{\nu}=\exp \left(-\int_{t}^{T} \theta\left(Y_{s}\right) d W_{s}^{1}-\int_{t}^{T} \nu_{s} d W_{s}^{2}\right) \quad \mathbb{P}_{t, y} \text {-a.s. }
$$

Let us now introduce the function

$$
\begin{aligned}
J(t, y, \eta, \nu) & :=\mathbb{E}_{t, y}\left[\left(D_{T}^{\eta}\right)^{1-\alpha}\left(Z_{T}^{\nu}\right)^{\alpha}\left(S_{T}^{0}\right)^{-\alpha}\right] \\
& =\mathbb{E}_{t, y}\left[\Delta_{T}^{\eta, \nu} \exp \left(\int_{t}^{T} q\left(Y_{s}, \eta_{s}, \nu_{s}\right) d s\right)\right]
\end{aligned}
$$

so that $J\left(0, Y_{0}, \eta, \nu\right)=\Lambda_{\eta, \nu}$ and $J(T, y, \eta, \nu)=1$. We will now use dynamic programming methods to solve the stochastic control problem with value function defined by

$$
V(t, y):=\sup _{\nu \in \mathcal{M}_{0}} \sup _{\eta \in \mathcal{C}} J(t, y, \eta, \nu)
$$

To this end, we first fix two controls $\eta \in \mathcal{C}$ and $\nu \in \mathcal{M}_{0}$. We can then define a new probability measure $P_{t, y}^{\eta, \nu} \sim \mathbb{P}_{t, y}$ by $d P_{t, y}^{\eta, \nu}=\Delta_{T}^{\eta, \nu} d \mathbb{P}_{t, y}$. According to (16), we have

$$
J(t, y, \eta, \nu)=E_{t, y}^{\eta, \nu}\left[\exp \left(\int_{t}^{T} q\left(Y_{s}, \eta_{s}, \nu_{s}\right) d s\right)\right] .
$$

There are two $P_{t, y}^{\eta, \nu}$-Brownian motions $W^{1, \eta, \nu}$ and $W^{2, \eta, \nu}$ such that

$$
\begin{aligned}
& d W_{s}^{1}=d W_{s}^{1, \eta, \nu}+\left((1-\alpha) \eta_{1 s}-\alpha \theta\left(Y_{s}\right)\right) d s \\
& d W_{s}^{1}=d W_{s}^{2, \eta, \nu}+\left((1-\alpha) \eta_{2 t}-\alpha \nu_{s}\right) d s .
\end{aligned}
$$

The parameter process $Y$ then satisfies the SDE

$$
d Y_{s}=d \widetilde{W}_{s}^{\eta, \nu}+\left\{g\left(Y_{s}\right)+\rho\left((1-\alpha) \eta_{1 s}-\alpha \theta\left(Y_{s}\right)\right)+\bar{\rho}\left((1-\alpha) \eta_{2 s}-\alpha \nu_{s}\right)\right\} d s,
$$

where $\widetilde{W^{\eta, \nu}}:=\rho W^{1, \eta, \nu}+\bar{\rho} W^{2, \eta, \nu}$ is a $P_{t, y}^{\eta, \nu}$-Brownian motion. Standard control theory [10] now suggests that the function $V$ is (formally) a solution to the Hamilton-Jacobi-Bellman 
(HJB) equation

$$
\begin{aligned}
0=v_{t}+\frac{1}{2} v_{y y} & +(g-\alpha \rho \theta) v_{y}+ \\
& \sup _{\nu \in \mathbb{R}} \sup _{\eta \in \Gamma}\left(\left[\rho(1-\alpha) \eta_{1}+\bar{\rho}\left((1-\alpha) \eta_{2}-\alpha \nu\right)\right] v_{y}+q(\cdot, \eta, \nu) v\right)
\end{aligned}
$$

with terminal condition

$$
v(T, y)=1
$$

This formal argument is made precise by the main result of this section:

Theorem 4.1 The function $V(t, y)$ is the unique bounded classical solution of the HJB equation (17)-(18).

The proof of this theorem will be prepared by two auxiliary lemmas, the first being a standard verification result. These lemmas will first be applied with the choice $I:=$ $[-M, M]$, which corresponds to restricting the control space for $\nu$ in (17). The fact that $I$ is compact will allow us to apply existence results for classical solutions $v^{I}$ of the corresponding HJB equation. An application of Lemma 4.3 will then guarantee that $v^{I}$ also solves the original HJB equation (17) provided that $M$ is large enough. Choosing $I:=\mathbb{R}$ in Lemma 4.2 will then yield the desired result. The proof of Theorem 4.1 will be given after the one of Lemma 4.3 .

Lemma 4.2 Let I be a nonempty real interval, which is either compact or equal to $\mathbb{R}$, and suppose that the HJB equation

$$
\begin{aligned}
0=v_{t}+\frac{1}{2} v_{y y}+(g-\alpha \rho \theta) v_{y}+ & \\
& \sup _{\nu \in I} \sup _{\eta \in \Gamma}\left(\left[\rho(1-\alpha) \eta_{1}+\bar{\rho}\left((1-\alpha) \eta_{2}-\alpha \nu\right)\right] v_{y}+q(\cdot, \eta, \nu) v\right)
\end{aligned}
$$

admits a bounded classical solution $v^{I}$ satisfying the terminal condition

$$
v^{I}(T, y)=1
$$

In case $I=\mathbb{R}$ we assume furthermore that $v^{I}$ is bounded away from 0 and has a bounded gradient. Then we have $v^{I}(t, y)=V^{I}(t, y)$, where

$$
V^{I}(t, y):=\sup _{\eta \in \mathcal{C}} \sup _{\nu \in \mathcal{M}^{I}} J(t, y, \eta, \nu)
$$

for $\mathcal{M}^{I}$ denoting the set of all I-valued $\nu \in \mathcal{M}_{0}$. In particular, we have uniqueness of bounded classical solutions. 
Proof: For $\nu \in I$ and $\eta \in \Gamma$ we define a differential operator $\mathcal{A}^{\eta, \nu}$ by

$$
\mathcal{A}^{\eta, \nu} f=f_{t}+\frac{1}{2} f_{y y}+\left(g+\rho((1-\alpha)-\alpha \theta)+\bar{\rho}\left(\left(1-\eta_{2}-\alpha \nu\right)\right) f_{y} .\right.
$$

Now let $\eta \in \mathcal{C}$ and $\nu \in \mathcal{M}^{I}$ be arbitrary controls. Then, by Itô's formula and (19), the function $v:=v^{I}$ satisfies

$$
\begin{aligned}
& d\left[e^{\int_{t}^{u} q\left(Y_{s}, \eta_{s}, \nu_{s}\right) d s} v\left(u, Y_{u}\right)\right] \\
& =e^{\int_{t}^{u} q\left(Y_{s}, \eta_{s}, \nu_{s}\right) d s}\left[v_{y}\left(u, Y_{u}\right) d \widetilde{W}_{u}^{\eta, \nu}+\left(\mathcal{A}^{\eta_{u}, \nu_{u}} v\left(u, Y_{u}\right)+q\left(Y_{u}, \eta_{u}, \nu_{u}\right) v\left(u, Y_{u}\right)\right) d u\right] \\
& \leq e^{\int_{t}^{u} q\left(Y_{s}, \eta_{s}, \nu_{s}\right) d s} v_{y}\left(u, Y_{u}\right) d \widetilde{W}_{u}^{\eta, \nu} .
\end{aligned}
$$

Letting $\tau_{n}:=\inf \left\{u \geq t|| v_{y}\left(u, Y_{u}\right) \mid \geq n\right\} \wedge T$, we hence get

$$
v(t, y) \geq E_{t, y}^{\eta, \nu}\left[e^{\int_{t}^{\tau_{n}} q\left(Y_{u}, \eta_{u}, \nu_{u}\right) d u} v\left(\tau_{n}, Y_{\tau_{n}}\right)\right] .
$$

Sending $n \uparrow \infty$ and using the boundedness of $v$ and $q^{+}$together with the terminal condition $v(T, \cdot)=1$, we obtain $v \geq V^{I}$. In particular, $v$ is strictly positive.

In order to prove the reverse inequality, note first that the supremum of the nonlinear term in (19) with respect to $\nu \in \mathbb{R}$ is attained in

$$
\widehat{\nu}=-\eta_{2}-\frac{\bar{\rho}}{1-\alpha} \cdot \frac{v_{y}}{v},
$$

which is always well-defined, due to the strict positivity of $v$. Hence, the supremum with respect to $\nu \in I$ is also attained, and we may find Markov controls

$$
\left(\eta^{*}, \nu^{*}\right) \in \underset{\nu \in I, \eta \in \Gamma}{\arg \max }\left\{\left[\rho(1-\alpha) \eta_{1}+\bar{\rho}\left((1-\alpha) \eta_{2}-\alpha \nu\right)\right] v_{y}(t, y)+q(y, \eta, \nu) v\right\},
$$

which by a measurable selection argument can be chosen as measurable functions $\eta^{*}(t, y)$, $\nu^{*}(t, y)$ of $t$ and $y$. Using the controls $\nu_{s}:=\nu^{*}\left(s, Y_{s}\right), \eta_{s}:=\eta^{*}\left(s, Y_{s}\right)$, we get an equality in $(22)$ and hence in (23).

Furthermore, we have the following estimates for the value function $V^{I}$.

Lemma 4.3 For a nonempty closed interval I containing the origin, let $V^{I}$ be the value function defined in (21). Then there exists a finite constant $K_{1}$ depending only on $\alpha, \theta, r$, $g$, and $\Gamma$ such that

$$
e^{-(T-t) K_{1}} \leq V^{I}(t, y) \leq e^{(T-t) K_{1}}
$$

Furthermore, $V^{I}$ is Lipschitz continuous, and its y-derivative satisfies

$$
\frac{\left|V_{y}^{I}(t, y)\right|}{V^{I}(t, y)} \leq K_{2} \quad \text { for a.e. } y
$$

where $K_{2}$ is a finite constant depending only on $\alpha, \theta, r, T, g$, and $\Gamma$. 
Proof: Clearly,

$$
q(y, \eta, \nu) \leq \alpha|r|_{\infty}=: K_{1}^{(+)}
$$

so that

$$
V^{I}(t, y) \leq \sup _{\eta \in \mathcal{C}} \sup _{\nu \in \mathcal{M}^{I}} \mathbb{E}_{t, y}\left[\Delta_{t}^{\eta, \nu}\right] \cdot e^{(T-t) K_{1}^{(+)}}=e^{(T-t) K_{1}^{(+)}}
$$

Moreover, for arbitrary $\eta \in \Gamma$,

$$
q(y, \eta, 0) \geq-\alpha(1-\alpha)\left(\max _{\eta \in \Gamma}|\eta|^{2}+|\theta|_{\infty}^{2}\right)-\alpha|r|_{\infty}=:-K_{1}^{(-)},
$$

so that

$$
V^{I}(t, y) \geq J(t, y, \eta, 0) \geq e^{-(T-t) K_{1}^{(-)}} .
$$

Taking $K_{1}:=K_{1}^{(+)} \vee K_{1}^{(-)}$thus gives the first assertion.

For the proof of the second one we fix $\eta \in \mathcal{C}$ and $\nu \in \mathcal{M}^{I}$. Let $Y$ and $\tilde{Y}$ denote solutions of the $\operatorname{SDE}(5)$ corresponding to initial values $Y_{t}=y$ and $\widetilde{Y}_{t}=\widetilde{y}$ under $\mathbb{P}_{t, y}$. Then

$$
\left|Y_{u}-\widetilde{Y}_{u}\right| \leq|y-\widetilde{y}|+\left|g^{\prime}\right|_{\infty} \int_{t}^{u}\left|Y_{s}-\tilde{Y}_{s}\right| d s,
$$

so that by Gronwall's lemma

$$
\left|Y_{u}-\widetilde{Y}_{u}\right| \leq|y-\widetilde{y}| \cdot e^{\left|g^{\prime}\right|_{\infty}(u-t)}
$$

Furthermore,

$$
\begin{aligned}
\left|\frac{\partial}{\partial y} q(y, \eta, \nu)\right| & =\left|\alpha(1-\alpha)\left(\eta_{1}+\theta(y)\right) \theta^{\prime}(y)-\alpha r^{\prime}(y)\right| \\
& \leq \alpha(1-\alpha)\left(\max _{\eta \in \Gamma}\left|\eta_{1}\right|+|\theta|_{\infty}\right)\left|\theta^{\prime}\right|_{\infty}+\alpha\left|r^{\prime}\right|_{\infty}=: L_{1} .
\end{aligned}
$$

Therefore,

$$
\begin{aligned}
& \left|e^{\int_{t}^{T} q\left(Y_{s}, \eta_{s}, \nu_{s}\right) d s}-e^{\int_{t}^{T} q\left(\widetilde{Y}_{s}, \eta_{s}, \nu_{s}\right) d s}\right| \\
& \leq e^{(T-t) K_{1}^{(+)}} \int_{t}^{T}\left|q\left(Y_{s}, \eta_{s}, \nu_{s}\right)-q\left(\widetilde{Y}_{s}, \eta_{s}, \nu_{s}\right)\right| d s \\
& \leq e^{T\left(K_{1}^{(+)}+\left|g^{\prime}\right|_{\infty}\right)} L_{1} T|y-\widetilde{y}|=: L_{2}|y-\widetilde{y}| .
\end{aligned}
$$

Next, let $\Delta^{\eta, \nu}$ and $\widetilde{\Delta}^{\eta, \nu}$ denote the stochastic exponentials in (16) corresponding to $Y$ and $\tilde{Y}$, respectively. Clearly,

$$
\Delta_{u}^{\eta, \nu}-\widetilde{\Delta}_{u}^{\eta, \nu}=\left(\Delta_{u}-\widetilde{\Delta}_{u}\right) \cdot \mathcal{E}\left(\int_{t}\left((1-\alpha) \eta_{2 s}-\alpha \nu_{s}\right) d W_{s}^{2}\right)_{u}
$$

where $\Delta$ and $\widetilde{\Delta}$ are the stochastic exponentials of the integrals with respect to $W^{1}$. Due to our assumption $\nu \in \mathcal{M}^{I} \subset \mathcal{M}_{0}$, the rightmost stochastic exponential is the density of a probability measure $\widehat{P} \sim \mathbb{P}_{t, y}$, under which the law of $W^{1}$ remains unchanged. Thus,

$$
\mathbb{E}_{t, y}\left[\left|\Delta_{u}^{\eta, \nu}-\widetilde{\Delta}_{u}^{\eta, \nu}\right|\right]=\widehat{E}\left[\left|\Delta_{u}-\widetilde{\Delta}_{u}\right|\right] \leq \widehat{E}\left[\left(\Delta_{u}-\widetilde{\Delta}_{u}\right)^{2}\right]^{1 / 2}=: \sqrt{\varphi(u)} .
$$


The function $\varphi$ satisfies

$$
\begin{aligned}
& \varphi(u) \leq \\
& \leq 2 \int_{t}^{u}\left\{\alpha^{2} \widehat{E}\left[\Delta_{s}^{2}\left(\theta\left(Y_{s}\right)-\theta\left(\widetilde{Y}_{s}\right)\right)^{2}\right]+\widehat{E}\left[\left(\Delta_{s}-\widetilde{\Delta}_{s}\right)^{2}\left((1-\alpha) \eta_{1 u}-\alpha \theta\left(\widetilde{Y}_{s}\right)\right)^{2}\right]\right\} d s \\
& \leq 2 T \alpha^{2}\left|\theta^{\prime}\right|_{\infty}^{2} e^{2\left|g^{\prime}\right|_{\infty} T} \widehat{E}\left[\Delta_{T}^{2}\right] \cdot|y-\widetilde{y}|^{2}+4\left((1-\alpha)^{2} \max _{\eta \in \Gamma}\left|\eta_{1}\right|^{2}+\alpha|\theta|^{2}\right) \int_{t}^{u} \varphi(s) d s .
\end{aligned}
$$

Since both $\theta$ and $\eta_{1}$ are bounded and $W^{1}$ is a $\widehat{P}$-Brownian motion, $\widehat{E}\left[\Delta_{T}^{2}\right]$ is bounded by a constant $c_{1}$, which only depends on $\theta$ and $\Gamma$. Hence, Gronwall's lemma and (27) yield

$$
\mathbb{E}_{t, y}\left[\left|\Delta_{u}^{\eta, \nu}-\widetilde{\Delta}_{u}^{\eta, \nu}\right|\right] \leq c_{2}|y-\widetilde{y}|
$$

where $c_{2}$ only depends on $\theta, \Gamma, \alpha$ and $T$.

Now we get from (26), (25), and (28) that

$$
\begin{aligned}
& |J(t, y, \eta, \nu)-J(t, \widetilde{y}, \eta, \nu)| \\
& \leq \mathbb{E}_{t, y}\left[\Delta_{T}^{\eta, \nu}\left|e^{\int_{t}^{T} q\left(Y_{s}, \eta_{s}, \nu_{s}\right) d s}-e^{\int_{t}^{T} q\left(\widetilde{Y}_{s}, \eta_{s}, \nu_{s}\right) d s}\right|\right]+\mathbb{E}_{t, y}\left[\left|\Delta_{T}^{\eta, \nu}-\widetilde{\Delta}_{T}^{\eta, \nu}\right| e^{\int_{t}^{T} q\left(\widetilde{Y}_{s}, \eta_{s}, \nu_{s}\right) d s}\right] \\
& \leq L_{2}|y-\widetilde{y}|+e^{T K_{1}^{(+)}} c_{2}|y-\widetilde{y}|=: \widetilde{K}_{2}|y-\widetilde{y}| .
\end{aligned}
$$

Thus, $V^{I}(t, \cdot)$ is Lipschitz continuous with constant $\widetilde{K}_{2}$, and the proof is completed by taking $K_{2}:=\widetilde{K}_{2} e^{K_{1} T}$.

Proof of Theorem 4.1: We first restrict the control space for $\nu$ to some bounded interval $I:=[-M, M]$. Then, from [10, Theorem IV.4.2 and Remark IV.3.3], there exists a bounded classical solution $v^{I}$ of the HJB equation (19)-(20). By Lemma 4.2, this solution is unique and corresponds to the value function $V^{I}$. As observed in (24), the supremum with respect to $\nu$ in (19) is achieved at

$$
\widehat{\nu}=-\eta_{2}-\frac{\bar{\rho}}{1-\alpha} \cdot \frac{V_{y}^{I}}{V^{I}}
$$

when this expression belongs to the set $]-M, M[$. Otherwise it will be achieved in the extremes of this set. By Lemma 4.3 we will have $|\widehat{\nu}|<M$ as soon as

$$
M>\max _{\eta \in \Gamma}\left|\eta_{2}\right|+\frac{\bar{\rho} K_{2}}{1-\alpha} .
$$

Hence the set $I$ in (19) can be substituted by $\mathbb{R}$, obtaining a bounded classical solution $v:=V^{I}$ to (19)-(20). Another application of Lemma 4.2 yields $v=V^{\mathbb{R}}=V$.

Corollary 4.4 The function $\log V(t, y)$ is the unique classical solution in $C_{b}^{1}([0, T] \times \mathbb{R}) \cap$ $C^{1,2}([0, T] \times \mathbb{R})$ of the HJB equation $(9)-(10)$. 
Proof: The nonlinear term in (17) can be simplified by computing first the infimum over $\nu \in \mathbb{R}$. To this end, we insert the optimal value (24) for $\nu$ back into the nonlinear term and obtain

$$
\begin{aligned}
& \sup _{\nu \in \mathbb{R}}\left(\left[\rho(1-\alpha) \eta_{1}+\bar{\rho}\left((1-\alpha) \eta_{2}-\alpha \nu\right)\right] v_{y}+q(\cdot, \eta, \nu) v\right) \\
& \quad=v\left[(1-\alpha) \rho \eta_{1} \frac{v_{y}}{v}-\frac{\alpha(1-\alpha)}{2}\left(\eta_{1}+\theta\right)^{2}+\bar{\rho} \eta_{2} \frac{v_{y}}{v}+\frac{\bar{\rho}^{2}}{2} \frac{\alpha}{1-\alpha}\left(\frac{v_{y}}{v}\right)^{2}-\alpha r\right]
\end{aligned}
$$

Thus, $V$ solves the HJB equation obtained by replacing the nonlinear term in (17) with the right-hand side of (29), and a simple computation shows that $w:=\log V$ solves (9). Conversely, if $w$ is a bounded classical solution of (9)-(10), then we can define $v:=e^{w}$ and reverse the chain of arguments to conclude that $v$ solves (17) and in turn is equal to $V$.

\section{Back to the primal problem}

In this section, we will complete the proof of Theorem 2.1 by using duality methods in obtaining a solution of the primal problem from the solution of the dual problem. To this end, recall from (14) and (15) that the terminal value of the optimal wealth process $X_{T}^{x, \widehat{\pi}}$ is given by

$$
X_{T}^{x, \widehat{\pi}}=I\left(\frac{\widehat{\lambda} Z_{T}^{\widehat{v}}}{D_{T}^{\widehat{\eta}} S_{T}^{0}}\right),
$$

where $I(y)=-\widetilde{U}^{\prime}(y)=y^{\alpha-1}, \widehat{\eta}=\eta^{*}\left(t, Y_{t}\right)$ and $\widehat{\nu}=\nu^{*}\left(t, Y_{t}\right)$ are optimal Markovian controls, and

$$
\widehat{\lambda}=\left(\frac{\Lambda_{\widehat{\eta}, \widehat{\nu}}}{x}\right)^{1 /(1-\alpha)} .
$$

By $\left[21\right.$, Theorem 2.6], the process $M_{t}:=X_{t}^{x, \widehat{\pi}} Z_{t}^{\widehat{\nu}} / S_{t}^{0}$ is a $\mathbb{P}$-martingale. Hence, (6) yields that

$$
\frac{d M_{t}}{M_{t}}=\widehat{\pi}_{t} \sigma\left(Y_{t}\right) d W_{t}^{1}+d Z_{t}^{\widehat{\nu}}=\left(\widehat{\pi}_{t} \sigma\left(Y_{t}\right)-\theta\left(Y_{t}\right)\right) d W_{t}^{1}-\widehat{\nu}_{t} d W_{t}^{2}
$$

where the computation simplifies by using the martingale property to conclude that all finite-variation terms must cancel out. On the other hand, by the Markov property,

$$
\begin{aligned}
M_{t} & =\mathbb{E}\left[M_{T} \mid \mathcal{F}_{t}\right]=\mathbb{E}\left[\left(\frac{\widehat{\lambda} Z_{T}^{\widehat{\nu}}}{D_{T}^{\widehat{\eta}} S_{T}^{0}}\right)^{\alpha-1} Z_{T}^{\widehat{\nu}} \frac{1}{S_{T}^{0}} \mid \mathcal{F}_{t}\right] \\
& =\frac{x}{\Lambda_{\widehat{\eta}, \widehat{\nu}}}\left(D_{t}^{\widehat{\eta}}\right)^{1-\alpha}\left(Z_{t}^{\widehat{\nu}}\right)^{\alpha}\left(S_{t}^{0}\right)^{-\alpha} J\left(t, Y_{t}, \widehat{\eta}, \widehat{\nu}\right) \\
& =\frac{x}{V\left(0, Y_{0}\right)}\left(D_{t}^{\widehat{\eta}}\right)^{1-\alpha}\left(Z_{t}^{\widehat{\nu}}\right)^{\alpha}\left(S_{t}^{0}\right)^{-\alpha} V\left(t, Y_{t}\right) .
\end{aligned}
$$

Thus, we get

$\frac{d M_{t}}{M_{t}}=(1-\alpha)\left(\widehat{\eta}_{1 t} d W_{t}^{1}+\widehat{\eta}_{2 t} d W_{t}^{2}\right)-\alpha\left(\theta\left(Y_{t}\right) d W_{t}^{1}+\widehat{\nu}_{t} d W_{t}^{2}\right)+\frac{V_{y}\left(t, Y_{t}\right)}{V\left(t, Y_{t}\right)}\left(\rho d W_{t}^{1}+\bar{\rho} d W_{t}^{2}\right)$, 
where the martingale property again significantly simplifies the computation. Comparing here and in (30) all terms involving $d W^{1}$ yields

$$
\widehat{\pi}_{t}=\frac{1}{\sigma\left(Y_{t}\right)}\left[(1-\alpha)\left(\widehat{\eta}_{1 t}+\theta\left(Y_{t}\right)\right)+\rho \frac{V_{y}\left(t, Y_{t}\right)}{V\left(t, Y_{t}\right)}\right]=\pi^{*}\left(t, Y_{t}\right)
$$

where

$$
\pi^{*}(t, y)=\frac{1}{\sigma(y)}\left[(1-\alpha)\left(\eta_{1}^{*}(t, y)+\theta(y)\right)+\rho \frac{V_{y}(t, y)}{V(t, y)}\right] .
$$

This completes the proof of Theorem 2.1.

Acknowledgement: Reaserch of D. H-H was supported by the project MathFi from INRIA Rocquencourt, France. Part of this work was done while the first author visited the Institute of Mathematics of the Berlin University of Technology. He wishes to thank for its support and hospitality

\section{References}

[1] Artzner, P., Delbaen, F., Eber, J.-M., Heath, D., Coherent measures of risk. Math. Finance 9 (1999), 203-228.

[2] Artzner, P., Delbaen, F., Eber, J., Heath, D., Ku, H. Coherent multiperiod riskadjusted values and Bellman's principle. Preprint, ETH Zürich, 2003.

[3] Bordigoni, G. Matoussi, A. Schweizer, M. A stochastic control approach to a robust utility maximization problem. To appear in Proceedings of Abel Symposium 2005, Springer.

[4] Burgert, C., Rüschendorf, L. Optimal consumption strategies under model uncertainty. Statist. Decisions 23 (2005), no. 1, 1-14.

[5] Castañeda-Leyva, N., Hernández-Hernández D. Optimal investment in incomplete financial markets with stochastic volatility. Contemp. Math. 336, 119-136 (2003).

[6] Castañeda-Leyva, N., Hernández-Hernández D. Optimal consumption-investment problems in incomplete markets with stochastic coefficients. SIAM J. Control Optim. 44, No. 4, pp. 1322-1344 (2005).

[7] Delbaen, F. The structure of m-stable sets and in particular of the set of riskneutral measures. To appear in: Seminaire Probab. 39 (2006).

[8] Epstein, L., Schneider, M. Recursive multiple-priors. J. Economic Theory 113 (2003), $1-31$

[9] Fleming, W., Hernández-Hernández, D. Tradeoff between consumption and investments in incomplete financial markets. Appl. Math. Optim. 52 (2005), 219-235.

[10] Fleming, W., Soner, M. Controlled Markov processes and viscosity solutions. Springer-Verlag, New York, 1993.

[11] Föllmer, H., Gundel, A. Robust projections in the class of martingale measures. To appear in Illinios J. Math. 
[12] Föllmer, H., Schied, A., Robust representation of convex measures of risk. In: Advances in Finance and Stochastics. Essays in Honour of Dieter Sondermann, SpringerVerlag, 2002, 39-56.

[13] Föllmer, H., Schied, A. Stochastic Finance: An Introduction in Discrete Time. Berlin: de Gruyter Studies in Mathematics 27 (2002). Second edition (2004).

[14] Gilboa, I., Schmeidler, D. Maximin expected utility with non-unique prior. J. Math. Econ. 18, 141-153 (1989).

[15] Hansen, L., Sargent, T. Robust control and model uncertainty. American Economic Review 91, 60-66 (2001).

[16] Kramkov, D., Schachermayer, W. The asymptotic elasticity of utility functions and optimal investment in incomplete markets. Ann. Appl. Probab. 9, no. 3, 904-950 (1999).

[17] Maccheroni, F., Marinacci, M., Rustichini, A. Ambiguity aversion, malevolent nature, and the variational representation of preferences. Preprint 2004.

[18] Müller, M. Market completion and robust utility maximization. PhD Thesis, Humboldt-Universität zu Berlin (2005).

[19] Quenez, M.-C. Optimal portfolio in a multiple-priors model. Seminar on Stochastic Analysis, Random Fields and Applications IV, 291-321, Progr. Probab., 58, Birkhäuser, Basel, 2004.

[20] Schied, A. Optimal investments for robust utility functionals in complete market models. Math. Oper. Research. 30, No. 3, 750-764 (2005).

[21] Schied, A. Optimal investments for risk- and ambiguity-averse preferences: a duality approach. Preprint, TU Berlin (2005).

[22] Schied, A., Wu, C.-T. Duality theory for optimal investments under model uncertainty. Stat. Decisions 23, No. 3, 199-217 (2005).

[23] Schmeidler, D. Subjective probability and expected utility without additivity. Econometrica 57, no. 3, 571-587 (1989).

Alexander Schied

Institute of Mathematics, MA 7-4

Berlin University of Technology

Strasse des 17. Juni 136

10623 Berlin, Germany

schied@math.tu-berlin.de
Daniel Hernández-Hernández

Centro de Investigación en Matemáticas

Apartado Postal 402

Guanajuato, Gto. C.P. 36000

México

dher@cimat.mx 


\section{SFB 649 Discussion Paper Series 2006}

For a complete list of Discussion Papers published by the SFB 649, please visit http://sfb649.wiwi.hu-berlin.de.

001 "Calibration Risk for Exotic Options" by Kai Detlefsen and Wolfgang K. Härdle, January 2006.

002 "Calibration Design of Implied Volatility Surfaces" by Kai Detlefsen and Wolfgang K. Härdle, January 2006.

003 "On the Appropriateness of Inappropriate VaR Models" by Wolfgang Härdle, Zdeněk Hlávka and Gerhard Stahl, January 2006.

004 "Regional Labor Markets, Network Externalities and Migration: The Case of German Reunification" by Harald Uhlig, January/February 2006.

005 "British Interest Rate Convergence between the US and Europe: A Recursive Cointegration Analysis" by Enzo Weber, January 2006.

006 "A Combined Approach for Segment-Specific Analysis of Market Basket Data" by Yasemin Boztuğ and Thomas Reutterer, January 2006.

007 "Robust utility maximization in a stochastic factor model" by Daniel Hernández-Hernández and Alexander Schied, January 2006.

SFB 649, Spandauer Straße 1, D-10178 Berlin http:/ / sfb649.wiwi.hu-berlin.de

This research was supported by the Deutsche Forschungsgemeinschaft through the SFB 649 "Economic Risk".

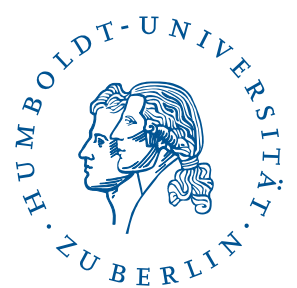

